\title{
Application Research of Indoor Plant Wall in Interior Decoration Design
}

\author{
Hu Xiaoli \\ Xi'an International University Shaanxi, China,710077
}

Keywords: Plant curtain wall; interior design; design method; evaluation;

\begin{abstract}
In the current architectural design, interior design has become an important content of concern. While creating a comfortable and comfortable living environment, the plant curtain wall has become an important way and means of decoration. In order to study the application of plant curtain wall in interior design, the paper analyzes the three aspects of design scheme, plant selection and design idea and uses AHP evaluation method to comprehensively evaluate the application of plant curtain wall in interior design. After analyzing the influencing factors of the interior design of the plant curtain wall, the excellent reference standard for constructing the plant wall is selected, which not only can achieve better interior decoration effect, but also enhance its artistic aesthetic value.
\end{abstract}

Plant curtain walls are also often referred to as green walls or ecological walls, mainly by imitating the state of plant growth in the natural environment, covering green plants on surface materials such as indoor walls, which are combined by green plants and planting facilities. A light and thin soilless cultivation technique established together has been widely used in interior design. At the same time, because of its special decorative performance and beautification function, it has become the main expression of environmental protection in interior design. It not only brings people a comfortable living environment, but also creates a vital natural beauty. Nowadays, many interior design studies focus on the structure of plant curtain wall, the selection of matrix and the growth of plants, but there is no relevant research and discussion on the application methods and application effects. Through the simple analysis of the design method of plant curtain wall, the selection of plant varieties and the application effect, it is expected to further explore and innovate the application of plant curtain wall in interior design ${ }^{[1]}$.

\section{The important role of plant landscape in interior design}

In the interior design, the plant landscape is a common application design. It produces a good design effect and use effect through scientific and reasonable design matching, which not only improves the design level, but also adds a sense of interior art. It has important practicalities. Sexual effect. First of all, the plant landscape can beautify the environment. Because the plant landscape itself has the natural beauty, it can show its vitality in the indoor environment. The use of interior design not only makes it more life-strength, but also serves as a leisure time for viewing plants. At the same time, it presents a variety of poses with different shapes and colors, enhancing the interior design effect, through organic the combination of the design makes the hardness of the space design significantly improved and adds a certain degree of softness. Secondly, the plant landscape can also play the role of purifying the air and also has a certain regulation effect on the air humidity. Under photosynthesis, the oxygen content in the indoor air can be enhanced and the humidity adjustment ability in winter is more significant. It is because the plant has the ability to decompose and treat harmful substances in the surrounding environment during the growth process. Under the decomposition, the harmful substances can be converted into carbon dioxide and water, so that the quality of the air can be comprehensively and effectively improved. Once again, the plant landscape has the effect of relieving stress, which may be unexpected, but again, because the plant itself is also alive and the vitality is quite tenacious, therefore, it plays an inspiring role in interior decoration. It can promote people to love life and nature more. At the same time, because of its delicate embellishment, people can always maintain a positive attitude in life and guide us to 
become the strong people of life. Undoubtedly, the indoor environment will affect our mood and make subtle changes in our mood. Therefore, through the decoration of the plant landscape, we can adjust our mental state, not only can we maintain a positive working condition, but also unlimited life enthusiasm. It can also alleviate the psychological pressure of people ${ }^{[2]}$.

\section{Application methods of plant curtain wall in interior design}

In the implementation of the design of the plant curtain wall, it must be based on the aesthetic design concept, through the artistic processing, determine the theme of the design, make reasonable arrangements, make the design level clear and adjust the basic shape and color matching and the overall style of interior design. In order to adapt to the impact of ecological factors, build a solid three-dimensional plant community. Referring to people's requirements and feelings about the shape, spatial extent and spatial privacy of the surrounding space environment, the interior space is continuously enriched by colors, materials, lighting, etc., so that they can satisfy the people while realizing the use value. Real needs, but also can provide users with a beautiful, comfortable, leisurely living atmosphere ${ }^{[3]}$.

\subsection{The artistic concept of design composition and the spatial form of the interior}

The compositional art methods of plant curtain walls include: key rendering, mediation and unity, comparison and imagination. Also pay attention to the proportion of composition. When constructing a picture design for a plant curtain wall in a room, the use of a large-area composition will make the screen of the curtain wall lean forward, so that the area of the room will be reduced. On the contrary, the area of the indoor space will be larger ${ }^{[4]}$.

\subsection{Color and indoor atmosphere}

When designing a plant curtain wall, the color combination rules include a combination of monochrome, contrasting colors and similar colors. In the combination of plant curtain wall color, green plants are generally used and other colors are used to make a reasonable combination and match to coordinate the colorful environment.

\subsection{The texture of the selected plant and the nature of the indoor environment}

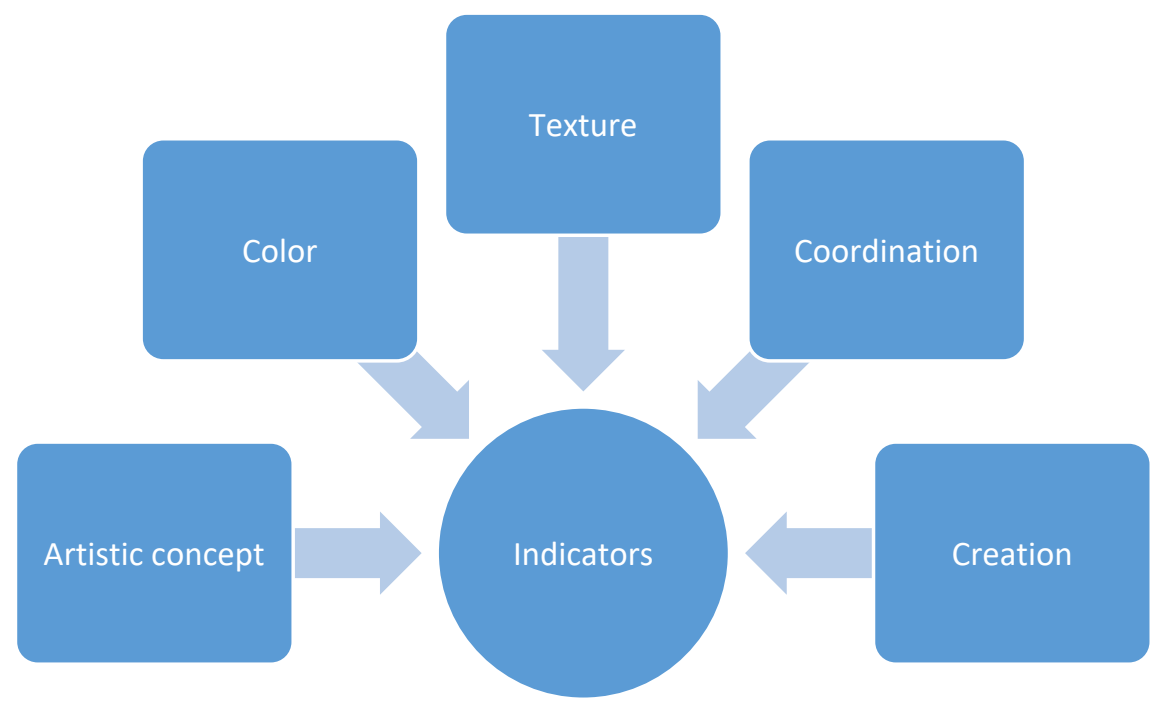

Figure 1: Five indicators that affect the interior decoration

When designing the plant curtain wall, plants with fine quality are selected as the foundation and the coarse plants are the core of the overall design and the medium-sized plants are used as 
auxiliary materials. Generally, the leaves of plants of fine quality are more regular and delicate, the branches are softer and softer, such as Tillandsia and moss; the plants of coarse type are thicker and wider than branches, such as Podocarpus and Turaea; The plants of the class belong to the combination of the above two types, creating an excellent plant curtain wall design with both characteristics and texture, which not only reflects the strong artistic rendering power, but also adds the unique feeling of the plant landscape. The five indicators that affect the interior decoration are shown in Figure 1.

\subsection{Coordination of plant morphology and indoor space}

In the design process of plant curtain wall, the plant form of some monomer forms should pay attention to the mutual matching of the overall morphology of the community. With exquisite and small leafy and multi-flowered plants, it is practically matched with some special-shaped plants to give embellishment. It is necessary to pay attention to the reasonable combination and matching of various plant forms.

\subsection{Creation of artistic conception and coordination of indoor space}

When designing the plant curtain wall, the artistic conception created by the plant is mostly based on the attributes and composition of the plant itself. At the same time, it combines with other indoor decoration facilities and lighting to create a preset atmosphere, cleverly associated with people. Willingness resonates with imagination.

\section{Application of plant curtain wall in interior design}

\subsection{Overview of the room}

The hotel used to host some foreign guests. The overall size of the plant's indoor curtain wall is about 200 square meters. This hotel requires that the plant curtain wall should have strong appreciation and safety and it should match the other settings and decoration styles in the room.

\subsection{Design theme and concept}

The hotel's plant curtain wall is based on the theme of returning to nature, extending the natural environment to the interior, the architecture and the natural environment are integrated, giving the interior a sense of vitality and fun. Reach people's love and love for the natural environment, giving people a comfortable and relaxing space ${ }^{[5]}$.

\subsection{Design plan}

The plant curtain wall of the pedestrian aisle is mainly in the form of a pattern, which is used to exchange a plant with a different plant to create a beautiful plant beauty. The plant curtain wall in the VIP room is mainly composed of natural forms and it is also equipped with some landscape stones to better show the calm and elegant atmosphere.

\subsection{Selection of various plants}

If the indoor plant curtain wall can be used for a long time, it is necessary to select the foliage plants that can survive for a long time and to decorate some plants with flowers. It is best to select plant varieties that can absorb the harmful substances in the room. In combination with a fragrant plant, it can make the interior fragrant and healthy. The hotel is an indoor space of shared and operated type. The requirements for the plant curtain wall can beautify the space and improve the environment and also attract guests. Therefore, the selection of plant varieties is more emphasis on appreciation, purification of air and the creation of a demand atmosphere.

\section{Evaluation of the effect of plant curtain wall in interior design}

\subsection{Determination of indoor plant curtain wall effect evaluation system}

According to the relevant standards of China and the related research on landscape evaluation, 
based on the content of indoor plant curtain wall design, some excellent examples of indoor plant curtain wall are analyzed and studied and the main influencing factors are compared and the evaluation criteria are compared. In order to select the principle and combine with the expert's insights, the specific evaluation criteria will be selected.

\subsection{Establishment of weights for evaluation indexes of indoor plant curtain walls}

After surveys of the masses and relevant professionals, with reference to the opinions of authoritative figures in the fields of landscape, plant and aesthetics, using Satie's 1 to 9 scale method to present, construct the trade-off matrix, the largest eigenvalue and the relative eigenvectors. Use a spreadsheet to calculate. By repeating the above method, the index weights of various evaluation subordinates can also be calculated. At this time, it can be seen from the results of the index weights that the environmental quality of the factor layer is the highest and the landscape effect is slightly worse. It is concluded that when designing the indoor plant curtain wall, the ecological benefits generated by the plant curtain wall are more concerned. The effect of appreciation and the need for decoration. The evaluation indicators are shown in Table 1.

Table 1: Evaluation indicators

\begin{tabular}{|c|c|c|c|}
\hline Index & Environment & Landscape & Healthy \\
\hline Weight & 0.4825 & 0.3164 & 0.2011 \\
\hline
\end{tabular}

\subsection{Establishment of the evaluation level of indoor plant curtain wall}

On the basis of the analysis of the actual case data, the overall evaluation standard level table of the indoor plant curtain wall was established with reference to some rules and regulations of the government on the indoor plant curtain wall, based on the authoritative evaluation results in the field and the scoring method of the comprehensive professional.

\section{Conclusion}

This paper combines the aspects of plant landscaping, landscape design and plant ecology. It summarizes the five aspects of composition, plant color, plant texture, plant shape and atmosphere and explores the design of indoor plant curtain wall. Ideas and methods. From the design of the theme concept, plant selection, design of the three aspects of the analysis and through the evaluation of the actual application of the indoor plant curtain wall of a star hotel, the indoor plant curtain wall design on the basis of the realization of functionality, Plant selection, not only should pay attention to the appreciation performance of the plant itself, but also have a good effect of purifying the environment. Under the analysis of the indoor plant curtain wall, the design principle of the indoor plant curtain wall is integrated. Through the analytic hierarchy process, the three aspects of the effect of the landscape, the benefits to the environment and the effects of the environment are elaborated. The evaluation system for the use of the curtain wall provides a reference for the use of the plant curtain wall.

\section{Acknowledgements}

This project was funded by Shaanxi provincial department of education and belongs to the project of construction of engineering quality technology evaluation system of indoor plant wall in Xi'An areas (16jk2179)

\section{References}

[1] Xixi Huang,Mingmin Zhang,Ling Lin,Zhigeng Pan,Rongzhao Li. The Design and Realization of Real-Time Texture Mapping and Collage in Virtual Home Decoration[P]. Digital Home (ICDH), 2012 Fourth International Conference on,2012. 
[2] Dánae Fiore. Art in time. Diachronic rates of change in the decoration of bone artefacts from the Beagle Channel region (Tierra del Fuego, Southern South America)[J]. Journal of Anthropological Archaeology,2011,30(4).

[3] Zi Che, Yizhou Lu. New decoration style in product design[P]. Computer-Aided Industrial Design \&amp; Conceptual Design, 2009. CAID \&amp; CD 2009. IEEE 10th International Conference on,2009.

[4] Shilian Xu, Jiaru Yang, Yanqin Wang, Haihong Liu, Junming Gao. Application of fractal art for the package decoration design[P]. Computer-Aided Industrial Design \&amp; Conceptual Design, 2009. CAID \&amp; CD 2009. IEEE 10th International Conference on,2009.

[5] Limei An, Haonan Tan, Jiankai Zhang, Yongrui Hou, Cuiqing Li. Energy-saving design in architectural decoration[P]. Multimedia Technology (ICMT), 2011 International Conference on,2011. 